\title{
Portable Biomarker Detection with Magnetic Nanotags
}

\author{
Drew A. Hall, Shan X. Wang, Boris Murmann \\ Stanford University \\ Department of Electrical Engineering \\ Stanford, CA, USA \\ \{drewhall, sxwang, murmann\}@stanford.edu
}

\begin{abstract}
This paper presents a hand-held, portable biosensor platform for quantitative biomarker measurement. By combining magnetic nanoparticle (MNP) tags with giant magnetoresistive (GMR) spin-valve sensors, the hand-held platform achieves highly sensitive (picomolar) and specific biomarker detection in less than 20 minutes. The rapid analysis and potential low cost make this technology ideal for point-ofcare (POC) diagnostics. Furthermore, this platform is able to detect multiple biomarkers simultaneously in a single assay, creating a promising diagnostic tool for a vast number of applications.
\end{abstract}

\section{INTRODUCTION}

Rapid and accurate measurement of biomarkers is a major challenge in diagnostic medicine. This is particularly apparent in point-of-care (POC) settings where the diagnostic platform is required to be portable and the analysis must be both highly sensitive and rapid. Currently, most diagnoses in developing countries and remote villages are made solely based on patient symptoms. In contrast, diagnoses are increasingly being driven by molecular testing in highly developed nations. To bring the diagnostic potential of the developed world into developing nations or the patient's own home, a highly sensitive, easy to use, and cost effective biosensing platform is needed. To this end, we have developed a portable biomarker platform called the NanoLab (Fig. 1) to address this pressing international need.

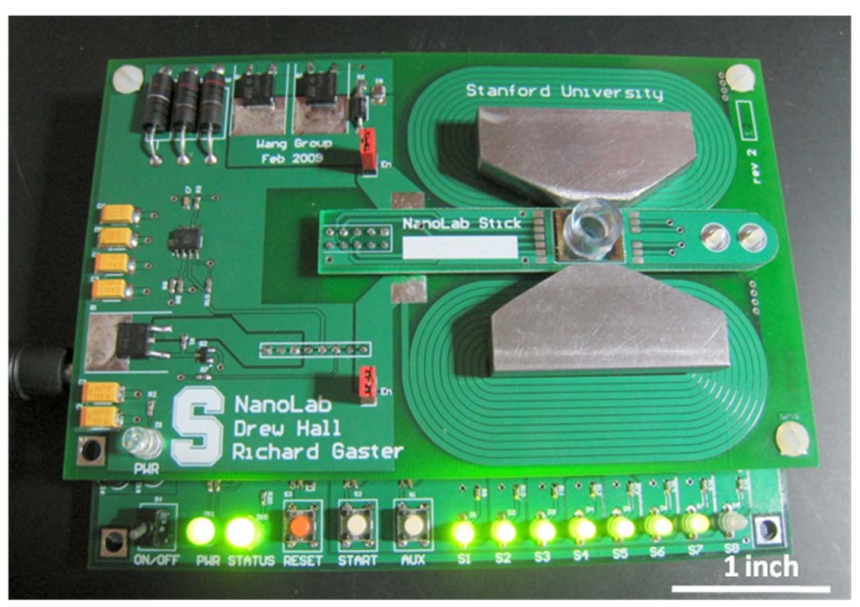

Figure 1. NanoLab test platform and disposable NanoLab Stick

\author{
Richard S. Gaster \\ Stanford University \\ Department of Bioengineering \\ Stanford, CA, USA \\ rgaster@stanford.edu
}

Biosensing utilizing magnetic tags and giant magnetoresistive (GMR) sensors was first demonstrated in 1998 by Baselt et al. [1]. Magnetic biosensors are very suitable to miniaturization and quantitative biomarker detection. In addition to the miniaturization potential, the main benefits of utilizing magnetic tags instead of colorimetric or fluorescent tags are the lower background (the samples naturally lack magnetic content) and the ability to manipulate the tags with a magnetic field [2]. The NanoLab provides rapid, multiplexed, and quantitative analysis of an entire panel of biomarkers simultaneously. While our previous work on GMR biosensing required a laboratory full of large, bulky, and expensive benchtop instrumentation [3-8], the NanoLab is handheld, battery powered, and fully integrated without the need for an external computer or PDA. The results are displayed to the user in real-time through an interface composed of colored light emitting diodes (LEDs) as shown in Fig. 1. The entire assay runs in an open well on a disposable NanoLab Stick in under 20 minutes, enabling true POC diagnostics.

\section{MAGNETIC IMMUNOASSAY}

The magnetic immunoassay is similar to the EnzymeLinked Immunosorbent Assay (ELISA), but replaces the fluorescent label with a magnetic tag. In this assay, a capture antibody, highly specific to a particular analyte (biomarker), is immobilized by covalently bonding the antibody to the surface of a magnetically responsive sensor. Upon introduction of several different analytes, each capture antibody selectively binds to a specific targeted analyte. Subsequently, a detection

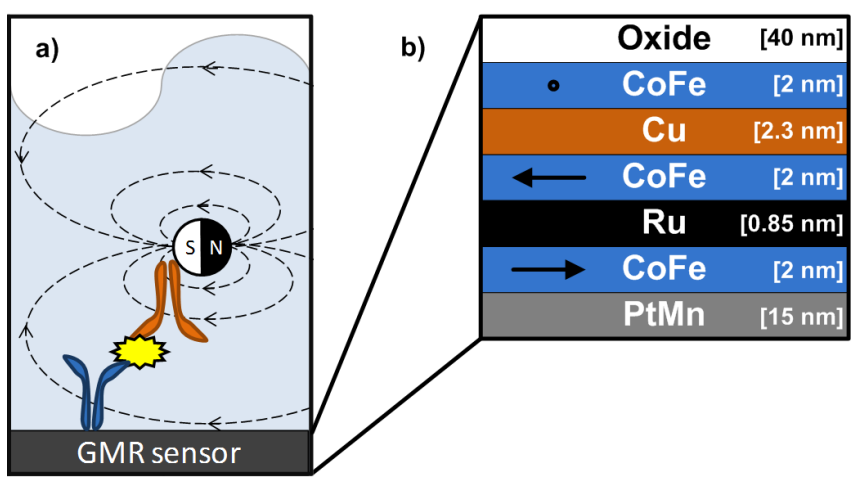

Figure 2. a) Illustration of magnetic immunoassay b) GMR spin-valve sensor

This work was supported in part by National Cancer Institute Grants 1U54CA119367 and 1U54CA143907, National Science Foundation (NSF) Grant ECCS-0801385-000, Defense Threat Reduction Agency Grant HDTRA1-07-1-0030-P00005, and the National Semiconductor Corporation. R.S.G. acknowledges financial support from Stanford MSTP. 
antibody complementary to the targeted analyte is added to the assay. Magnetic nanoparticle (MNP) tags complete the sandwich assay as shown in Fig. 2a [3]. The MNP tags are 50 $\mathrm{nm}$ in diameter and comprised of $10 \mathrm{~nm}$ superparamagnetic iron oxide cores embedded in a dextran shell. The underlying magnetically responsive sensors are proximity based sensors, detecting only the MNPs within approximately $150 \mathrm{~nm}$ of the sensor [4]. The sensors quantify the number of surface bound MNPs from the change in the local magnetic field. Multiplex detection is accomplished by immobilizing a different capture antibody on each of the individually addressable sensors.

While we demonstrate protein detection in this work, there is nothing limiting the platform to just protein detection. DNA, RNA, antibodies, and cells can also be detected by using an immobilized recognition molecule and a detection molecule tagged with a MNP.

\section{SENSORS}

GMR spin-valves (Fig. 2b) are well suited for POC diagnostics due to their small size, high sensitivity, low manufacturing cost, and their ability to be integrated with electronics on the same IC [8]. GMR spin-valves operate based on a quantum mechanical effect known as spindependent scattering. This effect causes the resistance of the sensor to change as a function of the external magnetic field. By monitoring miniscule changes in resistance caused by the MNP tags [5], we created a quantitative platform to accurately determine the number of biomarkers captured. A custom sensor die with 64 individually addressable GMR spin-valve sensors $(2.5 \mathrm{k} \Omega)$ was designed for this work [4], although only 8 of the sensors were used in the first iteration. The sensor die was wirebonded directly to a printed circuit board (PCB). A small well was fashioned on top of the sensors by gluing on a piece of Tygon tubing using two part epoxy. A dual inline-pin header was soldered to the end of the PCB to connect the disposable NanoLab Stick to the NanoLab test platform. The entire magnetic immunoassay runs in the small well (roughly $200 \mu \mathrm{L})$. An open well format was chosen for this application to reduce the cost and make the assay easy to run without requiring bulky external microfluidic pumps or valves.

\section{INTERFACE ELECTRONICS}

GMR spin-valve sensors are well known to exhibit high 1/f noise [9] prohibiting detection at DC. This is remedied by modulating the signal to a higher frequency by applying an alternating magnetic field (referred to as the tickling field) to the sensors. However, the time varying field also weakly couples onto the leads of the sensor making the system susceptible to mechanical motion where the stick is displaced inside of the test platform. This effect is removed by also modulating the excitation signal applied to the sensor. This double modulation scheme isolates the signal caused from the MNP and moves it to a higher frequency, away from the $1 / \mathrm{f}$ noise, improving the signal to noise ratio (SNR) $[10,11]$.

The entire signal path is shown in Fig. 3. An Analog Devices AD9833 direct digital synthesis (DDS) chip produces the modulated excitation signal. The output of the DDS chip is conditioned with a reconstruction filter, level shifted, amplified, and buffered prior to driving the sensor (Fig. 3a).

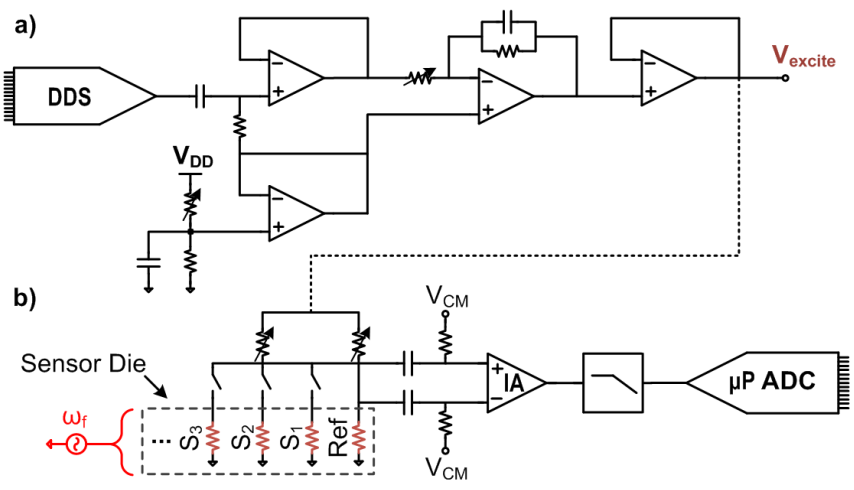

Figure 3. a) Signal synthesis schematic b) Sensor interface

The sensor is combined with a reference sensor (isolated from the MNP tags by thick epoxy) in a Wheatstone bridge configuration. An Analog Devices AD8221 instrumentation amplifier (IA) is AC coupled to the bridge and provides $60 \mathrm{~dB}$ of gain on the differential input. The signal is then digitized by the analog to digital converter (ADC) in the microprocessor after an antialiasing filter (Fig. 3b). The sensors are time domain multiplexed, sharing the signal path to save power and reduce the form factor, both of which are important considerations in POC settings. Digitally adjustable potentiometers are used in the bridge to accommodate process variations in the sensor die.

\section{INTEGRATED PlanAR ELECTROMAGNET}

The MNP tags require an external magnetic field to induce a magnetic moment and the sensors use the field to modulate the sensor response to a higher frequency. Previous work in our group has utilized large external Helmholtz coils [3-8, 11, 12] consuming hundreds of watts of power from a wall outlet. Miniaturizing the electromagnet to generate a uniform magnetic tickling field over the sensor array was a challenging aspect of this work. The electromagnet was realized out of $1.27 \mathrm{~mm}$ (50 mil) traces on a 4 layer PCB (Fig. 4b). The orientation of the current flowing through the coil alternates between clockwise and counter-clockwise to avoid the need for any cross-over traces that would reduce the number of available routing layers.

The tickling field is generated out of the plane (perpendicular to the PCB) and re-oriented by soft magnetic flux guides manufactured out of cold rolled steel. The flux guides concentrate the flux over a smaller region, acting as a form of passive amplification, and are used as heat sinks for the electromagnet. In addition to the flux guides above the coil, there are flux guides below the coil to close the flux loop and improve the efficiency. Due to the off-axis nature and the use of magnetic flux guides, analytical models are not tractable for design. Instead, finite element modeling (FEM) is needed to determine the required number of turns (11 turns per layer) and current, which depends on the field strength (Fig. 4c). Previous work [5] has shown that the optimum magnetic field for this particular combination of sensor and MNP is 25 Oe. However, the optimum is fairly shallow, allowing the field to be reduced without a significant loss in sensitivity. At a tickling field of 15 Oe ( $60 \%$ of the optimum), the signal per MNP only decreases $20 \%$ [5]. This small 
reduction in sensitivity allows the power consumption to be decreased dramatically. Fig. 4c shows the magnetic tickling field as a function of the applied AC current.

\section{POWER AMPLIFIER}

The power amplifier drives the electromagnet $(3 \Omega+300$ $\mu \mathrm{H})$ at $215 \mathrm{~Hz}$ with a $1 \mathrm{amp}$ peak current. A straightforward implementation of a class A amplifier is realized with an opamp driving a BJT Darlington pair (Fig. 4a). A sense resistor $\left(R_{s}\right)$ is part of the feedback loop to measure the current through the coil. The op-amp is powered from a regulated $5 \mathrm{~V}$ supply while the coil is supplied from an unregulated 11 volt $2100 \mathrm{mAh}$ rechargeable lithium ion polymer (LiPo) battery. To reduce the power consumption, the electromagnet is power cycled when it is not in use. Theoretically such a battery could last for almost 8.5 hours allowing 25 tests to be performed on a single charge. A diode $\left(D_{1}\right)$ clamps the back-EMF voltage across the electromagnet to protect the transistors when the power is abruptly switched off. The same circuit used to synthesize the signal driving the Wheatstone bridge (Fig. 3a) was replicated to drive the power amplifier.

\section{SIGNAL PROCESSING}

At the heart of the NanoLab is a Microchip microprocessor (dsPIC30F6012a) running at $80 \mathrm{MHz}$ (20 MIPS). The microprocessor has an integrated 12-bit ADC used to digitize the signals from the sensors. Furthermore, it communicates to the DDS chips via an integrated SPI bus. However, the primary reason for choosing a high-end microprocessor is for the heavy digital signal processing (DSP) algorithms that it performs. To extract the single tone from the spectrum with the double modulation scheme, a 113 tap digital FIR bandpass filter is applied to the incoming samples. The tap count was chosen after all of the code had been written such that it filled the remaining memory of the microprocessor to minimize the noise bandwidth of the extracted tone. The RMS value of the filter output is proportional to the magnetoresistance of the sensor and saved to an internal buffer. The sensors are scanned in a round robin fashion rotating from sensor 1 (S1) through sensor 8 (S8). For each sensor, the signal is acquired for 200 $\mathrm{ms}$ and the subsequent analysis takes $800 \mathrm{~ms}$. While the signal processing could be applied in real-time or overlapped with the data acquisition, the tasks are done sequentially to simplify the timing between the acquisition and the processing steps. This signal acquisition and processing is repeated continually throughout the duration of the test.

Prior to adding any MNP tags, several measurements are made and averaged to obtain the baseline magnetoresistance of each sensor. Every new data point (after the above signal processing steps) is compared to this baseline value to determine the change in magnetoresistance. The change in magnetoresistance is converted to an analyte concentration through pre-programmed concentration tables. The concentration tables have been quantized into four regions based on therapy regimen corresponding to undetectable, low, medium and high concentrations. The LED for each sensor is unlit, green, orange, or red corresponding to the threshold values of the concentration curve. This readout scheme requires no further analysis by the end-user. While this
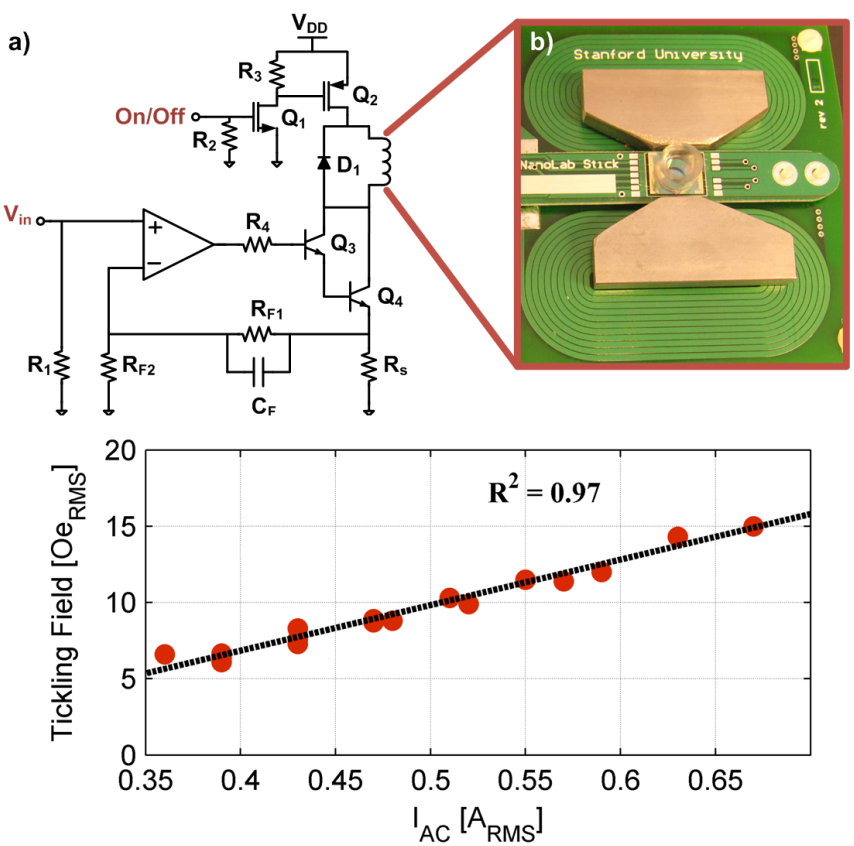

(c)

Figure 4. a) Power amplifier b) Planar electromagnet and flux guides c) Measured tickling field versus applied current

readout provides only semi-quantitative results, we also integrated the option to connect the device to a computer via a serial port to obtain fully quantitative real-time data.

\section{RESULTS}

We demonstrate detection of several spiked protein samples to verify the specificity, multiplexing ability, and reproducibility of the magnetic immunoassay. The biomarkers used for the validation tests were vascular endothelial growth factor (VEGF), carcinoembryonic antigen (CEA), and epithelial cell adhesion molecule (EpCAM). While these tumor markers are not specific to a particular disease state, they were chosen to highlight the generalizability of the platform. Bovine serum albumin (BSA) was included as a control chemistry to monitor the specificity of the assay and epoxy covered sensors to observe systematic errors with the electronics. Each of the disposable NanoLab Sticks was functionalized with VEGF (S1 and S2), CEA (S3 and S4), BSA (S5), epoxy (S6), and EpCAM (S7 and S8). The sample was incubated for 10 minutes prior to adding the MNP tags and the binding curves measured for an additional 10 minutes. More sensitive detection can be achieved with increased incubation time [3]. However, for most clinically significant concentration ranges, this is a sufficient compromise between incubation and readout time for rapid analysis.

In the first experiment, VEGF protein was spiked into PBS solution at $1 \mathrm{ng} / \mathrm{mL}(22 \mathrm{pM})$. The analytes for CEA and EpCAM were intentionally not added to monitor the nonspecific binding and cross-reactivity. Fig. 5a shows that after 4 minutes, when the MNP tags were added, only the two VEGF functionalized sensors responded, indicating that there is negligible nonspecific binding and no cross-reactivity. Furthermore, the duplicate sensors show evidence of the high reproducibility in the assay. 


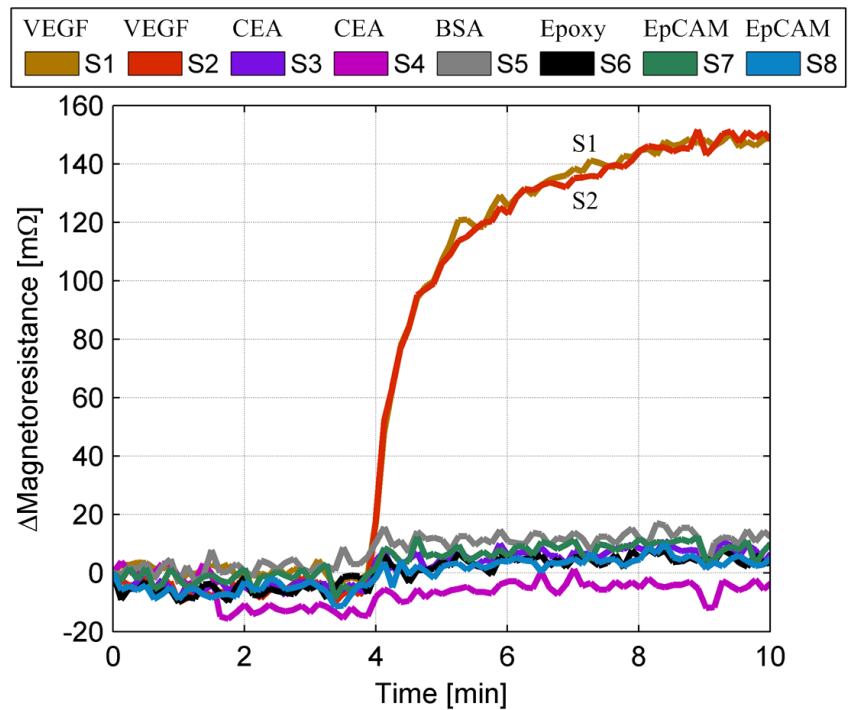

(a)

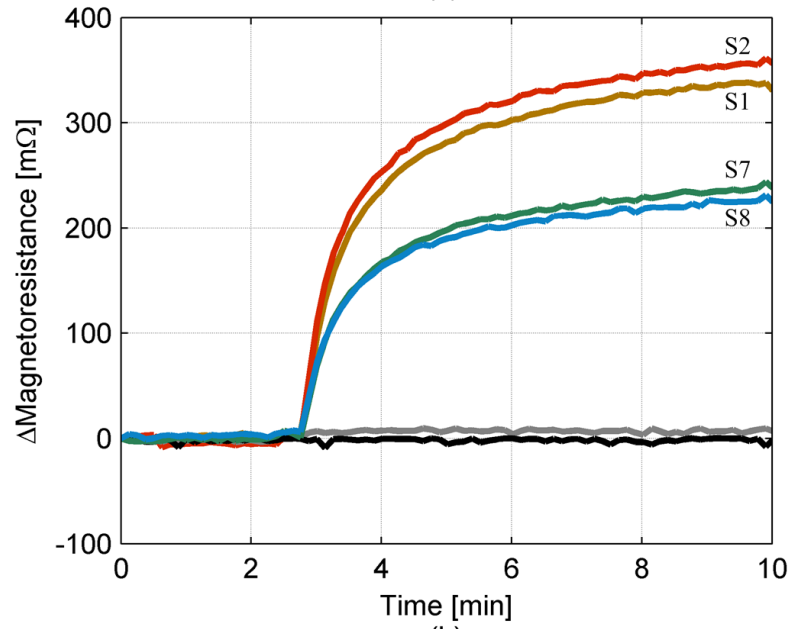

(b)

Figure 5. Change in magnetoresistance of GMR spin-valve sensor over time due to surface bound MNP for a) $1 \mathrm{ng} / \mathrm{mL}$ and b) $10 \mathrm{ng} / \mathrm{mL}$

In the second experiment, we demonstrate the multiplexing ability as well as the reproducibility. Fig. 5b shows detection of $10 \mathrm{ng} / \mathrm{mL}$ VEGF (222 pM) and EpCAM (286 pM) spiked into PBS. Prior to adding the MNP tags, the baseline signal is unresponsive and exhibits very little noise. After adding the tags, both the VEGF and EpCAM binding curves rise and quickly plateau to similar values, further demonstrating the reproducibility. Unfortunately, S3 and S4 were damaged due to a handling error during the preparation of the second NanoLab Stick. While all samples were performed in PBS buffer, these sensors have been shown to be equally effective for quantitative protein detection in a variety of media [3] making detection possible in serum, saliva, urine, etc. without any loss in signal.

\section{CONCLUSION}

Historically, medical diagnoses have relied heavily on molecular testing performed by expensive instrumentation confined to large laboratories. The new hand-held biomarker detection platform presented here shows promise for POC diagnostics and personal medicine. Detection down to low picomolar concentrations was demonstrated with a significant margin to quantify even lower concentrations of biomarkers. Throughout the design, a conscious effort was made to make the platform both cost effective and power efficient for portable applications. The NanoLab consumes an average of 3.7 watts from a rechargeable battery and weighs only $0.34 \mathrm{~kg}$ (0.75 lbs). This work provides a high-throughput and low cost point of care diagnostic device that will hopefully someday let individuals take healthcare into their own hands.

\section{ACKNOWLEDGMENT}

The authors thank Sebastian Osterfeld for GMR sensor design, Heng $\mathrm{Yu}$ for developing parts of the surface chemistry as well as other group members for their helpful discussions and advice.

\section{REFERENCES}

[1] D.R. Baselt, G.U. Lee, M. Natesan, S.W. Metzger, P.E. Sheehan, and R.J. Colton, "A biosensor based on magnetoresistance technology," Biosensors and Bioelectronics, vol. 13, pp. 731-739, Oct. 1998.

[2] H. Lee, A.M. Purdon, and R.M. Westervelt, "Manipulation of biological cells using a microelectromagnet matrix," Applied Physics Letters, vol. 85, 2004, pp. 1063-1065.

[3] R.S. Gaster, D.A. Hall, C.H. Nielsen, S.J. Osterfeld, H. Yu, K.E. Mach, R.J. Wilson, B. Murmann, J.C. Liao, S.S. Gambhir, and S.X. Wang, "Matrix-insensitive protein assays push the limits of biosensors in medicine," Nature Medicine, vol. 15, Nov. 2009, pp. 1327-1332.

[4] S.J. Osterfeld, H. Yu, R.S. Gaster, S. Caramuta, L. Xu, S. Han, D.A. Hall, R.J. Wilson, S. Sun, R.L. White, R.W. Davis, N. Pourmand, and S.X. Wang, "Multiplex protein assays based on real-time magnetic nanotag sensing," Proceedings of the National Academy of Sciences, vol. 105, pp. 20637-20640, Dec. 2008.

[5] D.A. Hall, R.S. Gaster, T. Lin, S.J. Osterfeld, S. Han, B. Murmann, and S.X. Wang, "GMR biosensor arrays: a system perspective," Biosensors and Bioelectronics, in press.

[6] L. Xu, H. Yu, M.S. Akhras, S. Han, S. Osterfeld, R.L. White, N. Pourmand, and S.X. Wang, "Giant magnetoresistive biochip for DNA detection and HPV genotyping," Biosensors and Bioelectronics, vol. 24, pp. 99-103, Sep. 2008.

[7] G. Li, S. Sun, R.J. Wilson, R.L. White, N. Pourmand, and S.X. Wang, "Spin valve sensors for ultrasensitive detection of superparamagnetic nanoparticles for biological applications," Sensors and Actuators A: Physical, vol. 126, pp. 98-106, Jan. 2006.

[8] S. Han, L. Xu, H. Yu, R. Wilson, R. White, N. Pourmand, and S. Wang, "CMOS integrated DNA microarray based on GMR sensors," Electron Devices Meeting, 2006. IEDM '06. International, 2006, pp. 14.

[9] R.J.M. van de Veerdonk, P.J.L. Belien, K.M. Schep, J.C.S. Kools, M.C. de Nooijer, M.A.M. Gijs, R. Coehoorn, and W.J.M. de Jonge, "1/f noise in anisotropic and giant magnetoresistive elements," Journal of Applied Physics, vol. 82, pp. 6152-6164, Dec. 1997.

[10] B. de Boer, J. Kahlman, T. Jansen, H. Duric, and J. Veen, "An integrated and sensitive detection platform for magneto-resistive biosensors," Biosensors and Bioelectronics, vol. 22, pp. 2366-2370, Apr. 2007.

[11] S. Han, H. Yu, B. Murmann, N. Pourmand, and S.X. Wang, "A highdensity magnetoresistive biosensor array with drift-compensation mechanism," Solid-State Circuits Conference, 2007. ISSCC 2007. Digest of Technical Papers. IEEE International, 2007, pp. 168-594.

[12] D.A. Hall, R.S. Gaster, S.J. Osterfeld, B. Murmann, and S.X. Wang, "GMR biosensor arrays: correction techniques for reproducibility and enhanced sensitivity," Biosensors and Bioelectronics, in press. 